\title{
Risk Factors for COVID-19 in Patients with Hypertension
}

\author{
Xinxin Wang $\mathbb{D}^{1}$, Haihua Zhang $\mathbb{D}^{1}$, Huan Du $\mathbb{D},{ }^{1}$ Ruina $M a \mathbb{D}^{1,2}$ \\ Yandong Nan $\mathbb{D}^{1,2}$ and Tao Zhang $\mathbb{D D}^{1,2}$ \\ ${ }^{1}$ Department of Pulmonary and Critical Care Medicine, Tangdu Hospital, Air Force Medical University, Xi'an, China \\ ${ }^{2}$ Wuhan Huoshenshan Hospital, Wuhan, China
}

Correspondence should be addressed to Yandong Nan; 13709205538@163.com and Tao Zhang; zhangtao19712020@126.com

Received 10 January 2021; Accepted 27 April 2021; Published 11 May 2021

Academic Editor: Mariam Arabi

Copyright ( 2021 Xinxin Wang et al. This is an open access article distributed under the Creative Commons Attribution License, which permits unrestricted use, distribution, and reproduction in any medium, provided the original work is properly cited.

Background. Hypertension, as the most common comorbidity for patients with coronavirus disease 19 (COVID-19), has resulted in cases with more severe symptoms and higher mortality. The risk factors associated with COVID-19 in patients with hypertension are unknown. Methods. All the available and confirmed patients with COVID-19 from February 3 to March 10, 2020, were enrolled from Huoshenshan Hospital, Wuhan, China. The demographic characteristics, clinical manifestations, laboratory data, radiological assessments, and treatments on admission were extracted and compared. Univariate and multivariate logistic regression methods were used to explore risk factors associated with COVID-19 in patients with hypertension and the severity of the cohort. Results. A total of 430 available patients with COVID-19 were enrolled in the study, including 151 eligible patients with COVID-19 and hypertension. After PSM analysis, 141 patients without hypertension and 141 cases with hypertension were well matched. Compared with cases without hypertension, patients with hypertension were more severe $(28.4 \%$ vs. $12.1 \%, p=0.001)$. In multivariate analysis, we found that neutrophil count (OR: $1.471 ; p=0.001)$, coronary heart disease (OR: $5.281 ; p=0.011$ ), and the level of $\mathrm{K}^{+}$(OR: $\left.0.273 ; p<0.001\right)$ were associated with patients with hypertension. In addition, the percentage of pulmonary infection volume was larger in cases with hypertension ( 4.55 vs. $5.8, p=0.017)$ and was a high risk factor for severe COVID-19 in patients with hypertension (OR: 1.084; $p<0.001$ ). Conclusion. On admission, coronary heart disease, neutrophil count, and the level of $\mathrm{K}^{+}$were associated with COVID-19 patients with hypertension. The percentage of the pulmonary infection volume was significantly larger in COVID-19 patients with hypertension and was a risk factor for COVID-19 severity of the cohort.

\section{Introduction}

The recently emerged severe acute respiratory syndrome coronavirus 2 (SARS-CoV-2), causing coronavirus disease 19 (COVID-19), poses a serious global health emergency because of its high pathogenicity and rapid international spread. Hypertension is the most common comorbidity for patients with COVID-19. A large number of studies have highlighted the overrepresentation of hypertension and the much higher fatality among patients with hypertension hospitalized with COVID-19 [1, 2]. Among 20982 patients confirmed to have COVID-19, $12.6 \%$ suffered from hypertension, which was the most frequent coexisting condition, and the overall proportion of hypertension was $39.75 \%$ in 406 deceased COVID-19 patients [3]. In another epidemiological study of an outbreak of COVID-19 from
China, the estimated prevalence of patients with hypertension was up to $12.8 \%$ among 44672 patients confirmed to have COVID-19, and the patients were more likely to develop serious symptoms, with a mortality rate of $6.0 \%$ [4]. In a report concerning 12226 patients who required hospital admission in 150 Spanish centers, hypertension (50.9\%) was the most common comorbidity and the preexisting condition of hypertension had an independent prognostic value for all-cause mortality in patients with COVID-19 [5].

SARS-CoV-2 is a new member of the beta-coronavirus genus and can recognize ACE2 on the lung alveolar epithelial cells as its host entry receptor, resulting in acute pulmonary infection [6]. Renin-angiotensin-aldosterone system (RAAS) inhibitors, including angiotensin-converting enzyme inhibitors (ACEIs) and angiotensin receptor blockers (ARBs), as one of the most important 
antihypertensive therapies, might upregulate the expression of ACE2 and thus facilitate the deterioration of patients' condition during the ongoing COVID-19 pandemic [7]. A large number of clinical studies and meta-analyses focused on antihypertensive therapies and have showed that RAAS inhibitors were beneficial to treat acute lung injury during severe acute respiratory syndrome coronavirus 2 (SARSCoV-2) infection. The risk factors associated with COVID19 patients with hypertension were still unknown.

In addition, chest radiological manifestation can reflect the actual lung infection objectively. As yet, no study uses quantifiable parameters on CT findings to assess the lung infection extent of COVID-19 patients with hypertension.

In the present study, we described the clinical characteristics of 282 COVID-19 patients with and without hypertension using the propensity score-matching (PSM) method to investigate the risk factors for COVID-19 in patients with hypertension. Besides, through comparing severe cases with nonsevere cases, 151 patients with COVID19 and hypertension were included to explore risk factors associated with the severity of COVID-19 in patients with hypertension. The lung infection percentage, which was calculated automatically using a deep learning-based software, was included. Our findings will provide guidance for the early administration of patients with COVID-19 and hypertension to prevent their further deterioration.

\section{Methods}

2.1. Participants. This single-center, retrospective study was conducted in Huoshenshan Hospital of Wuhan, in China, one of the makeshift hospitals dedicated to treating patients infected with the deadly coronavirus, which was constructed in one week, with a capacity of 1,000 beds, and formally delivered to military medics on February 2, 2020.

All patients with confirmed COVID-19 and hypertension, diagnosed according to the guidance provided by the fifth version of the guidelines issued by the National Health Commission of China on Diagnosis and Treatment of COVID-19 and the 2018 guidelines of the European Society of Hypertension (ESH) $[8,9]$ (Supplementary Table 1), were enrolled in our study, from February 3 to March 10, 2020. Pediatric patients and cases with an unclear history of the illness were excluded from the study. For discharged patients, we directly communicated with the patients or their families to ascertain their living status up to March 10, 2020.

The Ethics Commission of Huoshenshan Hospital approved this study. Informed consent was waived for this retrospective study.

2.2. Data Collection. The demographic characteristics, clinical symptoms, laboratory data, radiological assessments, and medications were extracted from electronic medical records and via telephone conversation for some unclear conditions, by a trained team of physicians. Two researchers then independently reviewed the collected data in case record forms and a third researcher judged any disagreements.
All the laboratory and radiological examinations were performed at Huoshenshan Hospital on admission, except for the nucleic acid test for SARS-CoV-2. From the 3rd to the 20th of February, respiratory specimens were sent to the local Centers for Disease Control and Prevention for SARSCoV-2 nucleic acid detection using next-generation sequencing or real-time reverse transcription PCR methods. After February 20, testing for SARS-CoV-2 nucleic acid was performed at Huoshenshan Hospital using real-time RTPCR methods.

Chest computed tomography (CT) was performed for all eligible inpatients. For the radiological examinations, not only were traditional descriptions including lesion shape, texture, and size reported, but also the infection volume ratio, which was calculated automatically using artificial intelligence (AI) software (United Imaging Intelligence, Shanghai, China), which could assess the severity of lung infection more precisely. There is a segmentation system in the software. After the baseline CT scans of patients were fed to the system, various metrics were computed to quantify the COVID-19 infection, including the percentages of infection in the whole lung (Figure 1) [10].

2.3. Definitions. Patients were divided into a severe group and a nonsevere group according to the fifth version of the guidelines issued by the National Health Commission of China on Diagnosis and Treatment of COVID-19 [8].

COVID-19 severity is classified as follows:

(1) Mild type: the clinical symptoms are mild with no abnormal radiological findings

(2) Moderate type: fever, cough, and other symptoms are presented with pneumonia on chest computed tomography

(3) Severe type: one of the following conditions is met: (1) respiratory distress and respiratory rate $\geq 30$ per min; (2) oxygen saturation on room air at rest $\leq 93 \%$; and (3) partial pressure of oxygen in arterial blood/ fraction of inspired oxygen $\leq 300 \mathrm{mmHg}$

(4) Critical type: one of the following conditions is met: (1) respiratory failure occurs and mechanical ventilation is required; (2) shock; and (3) patients with other organ dysfunction needing intensive care unit monitoring treatment

For limited critical cases with incomplete clinical data, severe, moderate, and mild types of COVID-19 patients were finally included in the study.

Hypertension was classified as Grade 1, Grade 2, and Grade 3 according to the 2018 guidelines of the European Society of Hypertension (ESH) [9] (Supplementary Table 1). Fever was considered as an axillary temperature above $37.2^{\circ} \mathrm{C}$. Shock was defined according to the Third International Consensus Definitions for Sepsis and Septic Shock (Sepsis-3) in 2016 [11]. The date of disease onset was defined as the day when the related symptoms appeared or abnormal chest radiological findings were observed. 


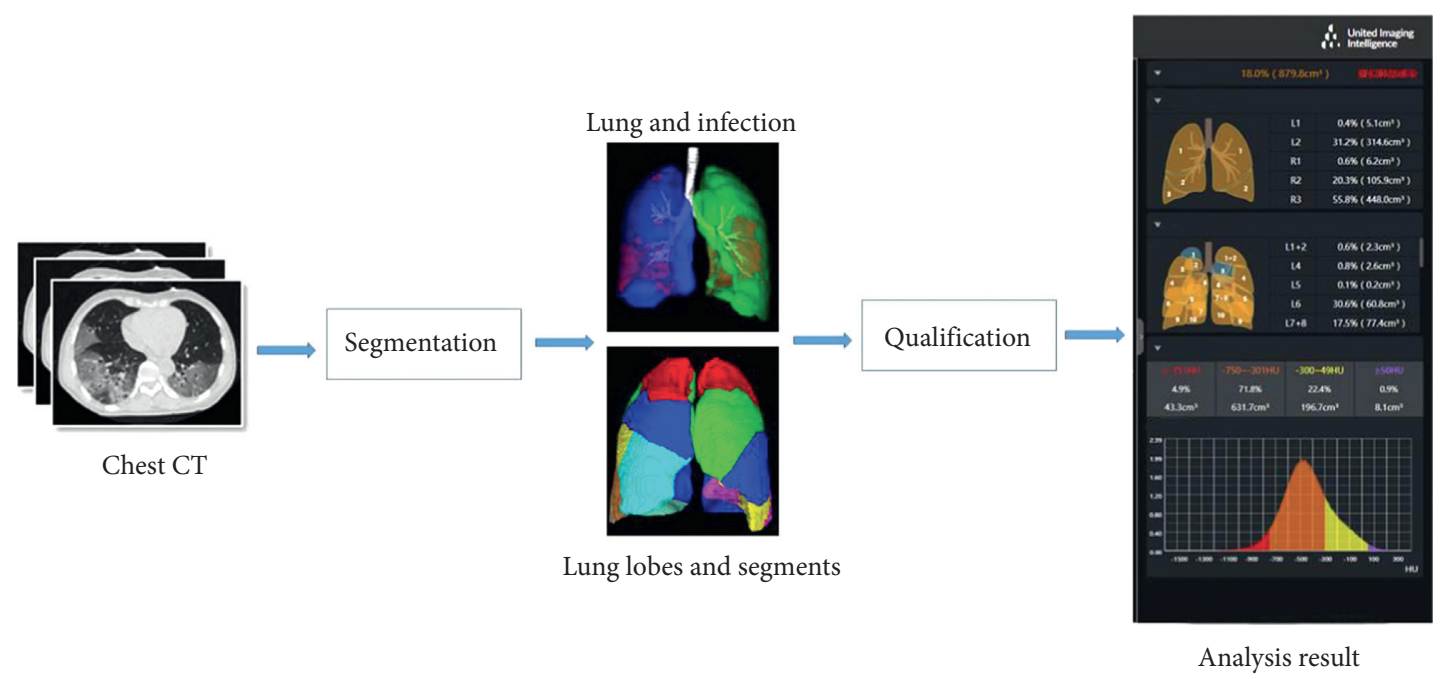

Figure 1: A flowchart for calculating the percentages of infection in the whole lung [10].

2.4. Statistical Analysis. In the statistical analysis, categorical variables expressed as proportions were compared using the chi-squared test and Fisher's exact test. Continuous variables, shown as the median with the interquartile range (IQR) values for nonnormally distributed data or as the means \pm standard deviation (SD) for normally distributed data, were compared using an independent group $t$-test or the nonparametric Mann-Whitney $U$ test, when appropriate.

PSM analysis was performed to minimize the effect of selection bias and to control for potential confounding factors. It is well known that older people have a higher likelihood to have hypertension. Age and sex matching between COVID-19 cohort with and without hypertension were conducted using a 1:1 matching protocol with caliper width $=0.2$ of the standard deviation of the propensity score.

Univariate and multivariate logistic regression methods were used to explore risk factors associated with the severity of COVID-19 in patients with hypertension. In the multivariate logistic regression, variables were excluded if their between-group differences were not significant from the univariate analysis. However, in the multivariate logistic regression of risk factors for severe cases with hypertension, considering the total number of patients with severe disease $(n=45)$, and to avoid overfitting, age, CK-MB (creatine kinase isoenzyme), ACEIs/ARBs treatment, platelet count, percentage of the infected region, and calcium ions were chosen for multivariate regression modeling using a backward stepwise model. The following factors were considered. Calcium channel blockers (CCB), as antihypertensive drugs, might influence the level of platelets and calcium ions. Coronary heart disease was the common complication for patients with hypertension, so $\mathrm{CK}-\mathrm{MB}$, as one of the cardiac biomarkers after troponin I, which was not significant from the univariate analysis, was included. In addition, previous studies had shown older age was a risk factor for poor outcome [12]. $p<0.05$ was considered statistically significant. All analyses were conducted using IBM SPSS Statistics v 21.0 (IBM Corp., Armonk, NY, USA).

\section{Results}

The final analysis included 151 eligible patients with hypertension and COVID-19, including 45 severe and 106 nonsevere cases, from 430 available patients confirmed to have COVID-19 admitted to Huoshenshan Hospital by March 10, 2020. Among them, 9 patients were excluded because they were suspected or pediatric cases, had missing medical information, duplicated records, or critical cases with limited medical records, especially CT scan. After oneto-one PSM analysis, 141 cases from 270 COVID-19 patients without hypertension and 141 COVID-19 patients with hypertension were respectively matched (Figure 2).

Compared with cases without hypertension, patients with hypertension were more severe $(28.4 \%$ vs. 12.1 , $p=0.001)$ and were more likely to have a comorbidity of diabetes $(19.1 \%$ vs. $9.9 \%, p=0.028)$, coronary heart disease (17.0\% vs. $6.4 \%, p=0.005)$, and cerebrovascular disease $(8.5$ vs. $2.1, p=0.017)$. Only $20.2 \%$ had exposure to the Huanan seafood market. The most common symptom was fever (76.2\%), followed by cough (70.2\%) and fatigue $(59.9 \%)$. Sputum (9.9\% vs. 5.0\%, $p<0.001)$, headache $(39.7 \%$ vs. $4.3 \%, p<0.001)$, and myalgia (37.6\% vs. $19.1 \%, p=0.001)$ were the most common symptoms at onset of illness for patients without hypertension (Table 1).

In terms of laboratory findings, compared with patients without hypertension, patients with hypertension showed a higher median white blood cell count (6.2 vs. 5.4, $p<0.001)$, especially the neutrophil count (3.3 vs. 4.4 ), but a lower level of $\mathrm{K}^{+}(4.2$ vs. $4.4, p=0.002)$. There were significant differences in the levels of ALP (68.1 vs. 74.6, $p=0.019)$, creatinine ( 64.6 vs. $68.1, p=0.013$ ), uric acid ( 255.5 vs. 286 , $p=0.034)$, and urea nitrogen (4.64 vs. 5.12, $p=0.003$ ) between the two groups of patients. On admission, 271 (96.1\%) of the patients showed bilateral involvement on chest CT images. The most common abnormalities for patients with hypertension were multiple mottling opacity (90.1 vs. 55.3, $p<0.001$ ) and typical ground-glass opacity (84.4 vs. 63.8, $p<0.001$ ). On admission, patients with 


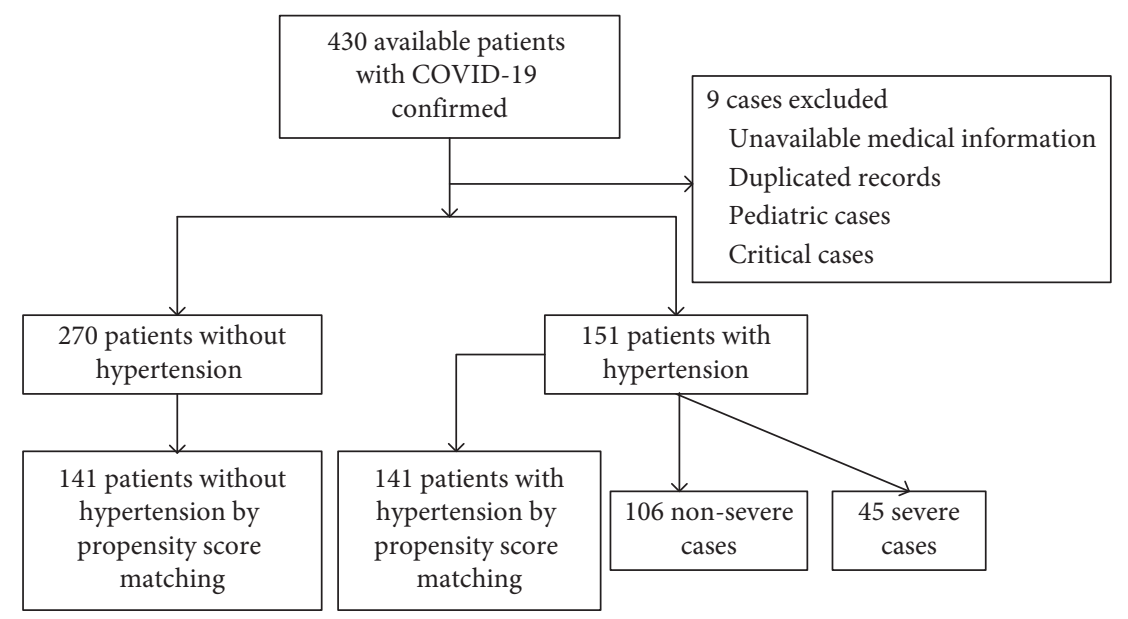

FIgURE 2: Flow diagram of patient screening and recruitment.

hypertension showed a larger percentage of the pulmonary infection volume than patients without hypertension (4.55 vs. 5.8, $p=0.017$ ) (Table 2 and Supplementary Table 2).

Among 151 patients with COVID-19 and hypertension, a total of 149 cases have primary hypertension, and two patients with nonsevere COVID-19 had secondary hypertension. The median length of hypertension was 10 years. However, 88 patients had suffered from hypertension for too long to recall the duration accurately. In terms of grade of hypertension, 11, 34, and 20 patients were classified as grade one, grade two, and grade three, respectively. Calcium channel blockers (CCBs) had been administered to 85 patients. Patients with nonsevere disease were more likely to require ACEIs/ARBs ( $24.5 \%$ vs. $8.9 \%, p=0.031)$, especially for ARBs (18.9\% vs. $2.2 \%, p=0.007)$, which was the second most popular antihypertensive treatment (Table 3).

Before the multivariate logistic analysis, univariate analysis was performed to screen out the appropriate variables. After univariate analysis, diabetes, coronary heart disease, cerebrovascular disease, decreased platelet count and calcium ions, elevated white blood cell count, neutrophil count, ALP, urea nitrogen, uric acid, creatinine, and decreased $\mathrm{K}^{+}$had effects on COVID-19 in patients with hypertension. In multivariate analysis, $\mathrm{We}$ found that neutrophil count (OR: $1.471, p=0.001$ ), coronary heart disease (OR: 5.281, $p=0.011$ ), and the level of $\mathrm{K}^{+}(\mathrm{OR}$ : 0.273, $p<0.001$ ) were high risk factors for COVID-19 in patients with hypertension (Tables 4 and 5).

In univariate analysis for 151 patients with hypertension, age, ACEIs/ARBs, lymphopenia, leucocytosis, percentage of the pulmonary infection volume, decreased platelet count and calcium ions, and elevated white blood cell count, neutrophil count, lactate dehydrogenase (LDH), myoglobin, D-dimer, procalcitonin (PCT), and C-reactive protein, AST, albumin, and CK-MB were explored for their association with severe COVID-19 in patients with hypertension (Supplementary Table 3). We found that the percentage of pulmonary infection volume was a high risk factor for severe COVID-19 in patients with hypertension (OR: 1.084, $p<0.001$ ) (Table 6).

\section{Discussion}

Hypertension is the most common coexisting medical condition for patients with COVID-19 and is associated with higher mortality. Hypertension usually can mediate target organ damage or cardiovascular disease, which may be determinant factors for COVID-19. Coronary heart disease is the main complication of hypertension. It was reported that cardiac injury was an independent predictive factor for the death of patients with COVID-19 [13]. Patients with preexisting cardiovascular diseases were more susceptible to COVID-19-induced heart injury [14]. In the present study, we also found that coronary heart disease was associated with COVID-19 patients with hypertension. However, there was no difference between patients with hypertension and those without hypertension for high-sensitivity troponin I, which is a specific cardiac indicator, as well as myoglobin and creatine kinase isoenzymes, as cardiac biomarkers. ACE2, as a monocarboxypeptidase glycoprotein and the entry receptor for SARS-CoV-2, was also expressed in the kidney [15]. In one study with 193 COVID-19 patients at hospital admission, more patients had higher rates of AKI, and COVID-19 patients with AKI had a significantly higher mortality risk [16]. In our study, creatinine and urea nitrogen were found to be higher in patients with hypertension, but in univariate analysis, was not associated with COVID-19 cases with hypertension. Our results demonstrated the percentage of pulmonary infection volume was different between COVID-19 patients with hypertension and cases without hypertension, and multivariate regression showed that the percentage of pulmonary infection volume was associated with the severity of COVID-19 in patients with hypertension.

Chest radiological manifestation can reflect the actual lung infection objectively. Usually, a computed tomography scoring system including radiographic features and distributions in two dimensions, which are finished manually, is used to assess the extent of pulmonary pathology in COVID19 patients $[17,18]$. While the scoring system can reflect the CT findings, a quantifiable parameter was still needed. To the best of our knowledge, this is the first report to analyze the 
TABle 1: Demographic and clinical characteristics of 282 patients with COVID-19.

\begin{tabular}{|c|c|c|c|c|}
\hline Clinical characteristics & All $(n=282)$ & $\begin{array}{l}\text { Nonhypertension } \\
\quad(n=141)\end{array}$ & $\begin{array}{l}\text { Hypertension } \\
\quad(n=141)\end{array}$ & $p$ value \\
\hline Age, median (IQR), years & $67(62-71)$ & $67(62-71)$ & $5.8(1.3-12.3)$ & 0.491 \\
\hline \multicolumn{5}{|l|}{ Sex } \\
\hline Female & $132(46.8)$ & $64(45.4)$ & $68(48.2)$ & 0.633 \\
\hline Male & $150(53.2)$ & $77(54.6)$ & $73(51.8)$ & \\
\hline Severe & $57(20.2)$ & $17(12.1)$ & $40(28.4)$ & 0.001 \\
\hline Nonsevere & $225(79.8)$ & $124(87.9)$ & $101(71.6)$ & \\
\hline Exposure history & $57(20.2)$ & $24(17.0)$ & $33(23.4)$ & 0.011 \\
\hline Nonexposure history & $119(42.2)$ & $28(19.9)$ & $91(64.5)$ & \\
\hline Smoking & $24(8.5)$ & $12(8.5)$ & $12(8.5)$ & 1 \\
\hline \multicolumn{5}{|l|}{ Comorbidities } \\
\hline Diabetes & $41(14.5)$ & $14(9.9)$ & $27(19.1)$ & 0.028 \\
\hline Carcinoma & $5(1.8)$ & 0 & $5(3.5)$ & 0.075 \\
\hline Coronary heart disease & $33(11.7)$ & $9(6.4)$ & $24(17.0)$ & 0.005 \\
\hline Cerebrovascular disease & $15(5.3)$ & $3(2.1)$ & $12(8.5)$ & 0.017 \\
\hline Chronic pulmonary disease & $5(1.8)$ & $5(3.5)$ & 0 & 0.071 \\
\hline Kidney disease & $10(3.5)$ & $4(2.8)$ & $6(4.3)$ & 0.52 \\
\hline Others & $51(18.1)$ & $26(18.4)$ & $25(17.7)$ & 0.877 \\
\hline \multicolumn{5}{|l|}{ Symptoms } \\
\hline Fever & $215(76.2)$ & $113(80.1)$ & $102(72.3)$ & 0.124 \\
\hline Cough & $19870.2)$ & $106(75.2)$ & $92(65.2)$ & 0.068 \\
\hline Pharyngodynia & $5(1.8)$ & $3(2.1)$ & $2(5.0)$ & 1 \\
\hline Sputum & $21(7.4)$ & $14(9.9)$ & $7(5.0)$ & $<0.001$ \\
\hline Wheezing & $92(32.6)$ & $51(36.2)$ & $41(29.1)$ & 0.204 \\
\hline Shortness of breath & $58(20.6)$ & $26(18.4)$ & $32(22.7)$ & 0.377 \\
\hline Nausea or vomiting & $6(2.1)$ & $5(3.5)$ & 10.7() & 0.216 \\
\hline Abdominal pain & $2(0.7)$ & $1(0.7)$ & $1(0.7)$ & 1 \\
\hline Diarrhea & $16(5.7)$ & $9(6.4)$ & $7(5.0)$ & 0.607 \\
\hline Chest distress & $51(18.1)$ & $27(19.1)$ & $24(17.0)$ & 0.643 \\
\hline Flusteredness & $5(1.8)$ & $3(2.1)$ & $2(1.44)$ & 1 \\
\hline Fatigue & $169(59.9)$ & $88(62.4)$ & $81(57.4)$ & 0.395 \\
\hline Headache & $62(30.0)$ & $56(39.7)$ & $6(4.3)$ & $<0.001$ \\
\hline Myalgia & $80(28.4)$ & $53(37.6)$ & $27(19.1)$ & 0.001 \\
\hline Dizziness & $22(7.8)$ & $14(9.9)$ & $8(5.7)$ & 0.183 \\
\hline Anorexia & $11(3.9)$ & $7(5.0)$ & $4(2.8)$ & 0.356 \\
\hline Rhinobyon & $3(1.1)$ & $1(0.7)$ & $2(1.4)$ & 1 \\
\hline \multicolumn{5}{|l|}{ Vital signs (IQR) } \\
\hline Respiratory rate, median (IQR), breaths per min & $20(19-21)$ & $20.00(19.50-21.00)$ & $20(19-22)$ & 0.867 \\
\hline Heart rate, median (IQR), beats per min & $84(78-90)$ & $84.00(78.00-91.50)$ & $82(78-90)$ & 0.37 \\
\hline Temperature, median (IQR), ${ }^{\circ} \mathrm{C}$ & $36.6(36.4-36.9)$ & $36.60(36.40-36.90)$ & $36.6(36.4-36.90$ & 0.941 \\
\hline Systolic blood pressure & $\begin{array}{c}130.00 \\
(120.00-139.75)\end{array}$ & $129.50(120.00-136.25)$ & $130(121-144)$ & 0.152 \\
\hline Diastolic blood pressure & $78.00(72.00-85.00)$ & $78.0(70.75-82.0)$ & $79.0(72.75-87.25)$ & 0.055 \\
\hline $\begin{array}{l}\text { Time from illness onset to admission, (IQR), } \\
\text { days }\end{array}$ & $14.00(10.00-18.00)$ & $14.0(10.0-17.0)$ & $14.0(10.0-19.5)$ & 0.447 \\
\hline
\end{tabular}

IQR: interquartile range.

percentage of pulmonary infection volume of patients with COVID-19 and hypertension in the hospital. In our study, the lung infection percentage was introduced to assess lung injury in three dimensions, which was calculated automatically using a deep learning-based software, instead of the rough estimate of the infection area obtained from traditional CT. SARS-CoV-2 was more likely to damage the lung tissue of patients with hypertension, leading to increased numbers of severe cases.

Recent understanding of the dysregulation immune system in hypertension may provide a possible explanation why hypertension is potentially associated with a more severe course of COVID-19. SARS-CoV-2 induces the overproduction of proinflammatory cytokines, including interleukin-6 (IL-6), IL-2, IL-7, and tumour necrosis factor$\alpha$ (TNF- $\alpha)$, resulting in a "cytokine storm" of overactivated neutrophils, monocyte, and lymphocytes [2]. In experimental and clinical observations, patients with hypertension showed inflammatory dysregulation. Clinical hypertension is undoubtedly associated with immune activation. Increased numbers of central memory CD8+ T cells, which can produce TNF, were found in patients with hypertension 
TABLE 2: Laboratory and radiographical findings of 282 patients with COVID-19 on admission.

\begin{tabular}{|c|c|c|c|c|}
\hline Clinical characteristics & All $(n=282)$ & Nonhypertension $(n=141)$ & Hypertension $(n=141)$ & $p$ value \\
\hline \multicolumn{5}{|l|}{ Laboratory findings, median (IQR) } \\
\hline White blood cell count, $\times 10^{9} / \mathrm{L}$ & $5.80(4.50-7.00)$ & $5.40(4.10-6.80)$ & $6.2(5.2-7.8)$ & $<0.001$ \\
\hline Neutrophil count, $\times 10^{9} / \mathrm{L}$ & $3.79(2.80-5.28)$ & $3.28(2.37-4.95)$ & $4.42(3.11-5.89)$ & $<0.001$ \\
\hline Monocyte count, $\times 10^{9} / \mathrm{L}$ & $0.44(0.33-0.57)$ & $0.42(0.32-0.57)$ & $0.45(0.35-0.57)$ & 0.746 \\
\hline Lymphocyte count, $\times 10^{9} / \mathrm{L}$ & $1.18(0.84-1.65)$ & $1.23(0.91-1.65)$ & $1.15(0.77-1.64)$ & 0.219 \\
\hline Haemoglobin, g/L (mean \pm SD) & $124.00(113.00-133.00)$ & $123.50(112.00-131.00)$ & $124(114-135)$ & 0.214 \\
\hline Platelet count, $\times 10^{9} / \mathrm{L}$ & $254.65(160.31-348.99)$ & $262.47(173.25-351.69)$ & $246.73(147.77-345.69)$ & 0.165 \\
\hline Eosinophil count, $\times 10^{9} / \mathrm{L}$ & $0.07(0.03-0.12)$ & $0.06(0.03-0.11)$ & $0.07(0.01-0.12)$ & 0.856 \\
\hline Basophils count, $10^{9} / \mathrm{L}$ & $0.01(0.01-0.02)$ & $0.01(0.01-0.02)$ & $0.01(0.01-0.02)$ & 0.772 \\
\hline C-reactive protein, $\mathrm{mg} / \mathrm{L}$ & $6.03(1.99-36.53)$ & $5.19(1.72-18.85)$ & $8.2(2.62-46.32)$ & 0.067 \\
\hline IL-6, pg/ml & $4.91(2.38-15.62)$ & $5.69(2.62-96.88)$ & $4.82(2.38-1.64)$ & 0.534 \\
\hline $\mathrm{PCT}, \mathrm{ng} / \mathrm{ml}$ & $0.05(0.03-0.20)$ & $0.05(0.03-0.07)$ & $0.06(0.03-0.41)$ & 0.438 \\
\hline ALT, IU/L ${ }^{\mathrm{a}}$ & $25.35(17.18-42.45)$ & $25.2(17.75-39.25)$ & $25.6(16.75-48.25)$ & 0.836 \\
\hline AST, IU/L & $22.50(16.8-31.65)$ & $21.9(16.25-28.43)$ & $23.4(17.5-36.0)$ & 0.135 \\
\hline Albumin, $\mathrm{g} / \mathrm{L}$ & $34.86(30.85-38.87)$ & $35.17(31.52-38.82)$ & $34.53(30.19-38.87)$ & 0.2 \\
\hline Total bilirubin, umol/ $/ \mathrm{L}^{\mathrm{a}}$ & $62.59(56.12-69.06)$ & $62.62(56.13-69.11)$ & $62.56(56.09-69.03)$ & 0.947 \\
\hline$\gamma$-Glutamyltransferase, IU/L & $28.90(20.20-57.00)$ & $27.9(18.7-44.0)$ & $32.8(20.5-67.40$ & 0.107 \\
\hline $\mathrm{ALP}, \mathrm{IU} / \mathrm{L}^{\mathrm{a}}$ & $71.50(58.20-91.50)$ & $68.1(56.73-83.55)$ & $74.6(60.25-96.2)$ & 0.019 \\
\hline Creatine kinase, IU/L & $46.80(33.65-71.70)$ & $49.3(33.6-71.30$ & $44.6(33.58-73.4)$ & 0.919 \\
\hline $\mathrm{LDH}, \mathrm{IU} / \mathrm{L}$ & $206.90(174.25-273.50)$ & $198.95(171.88-257.70)$ & $214.6(178.6-288.55)$ & 0.302 \\
\hline CK-MB, IU/L & $9.00(7.30-12.90)$ & $8.70(7.25-12.45)$ & $9.4(7.3-13.2)$ & 0.35 \\
\hline Myoglobin, ng/ml & $10.23(4.87-20.77))$ & $9.67(4.97-21.98)$ & $11.56(4.81-20.32)$ & 0.652 \\
\hline Hypersensitive troponin $\mathrm{I}, \mathrm{ng} / \mathrm{ml}$ & $0.01(0.01-0.02)$ & $0.01(0.01-0.18)$ & $0.01(0.01-0.02)$ & 0.729 \\
\hline $\mathrm{BNP}, \mathrm{pg} / \mathrm{ml}$ & $12.9(0.01-62.59)$ & $0.01(0.01-43.35)$ & $20.4(0.01-66.24)$ & 0.065 \\
\hline Creatinine, umol/ $\mathrm{L}^{\mathrm{a}}$ & $67.20(56.70-79.95)$ & $64.60(55.10-74.50)$ & $68.1(57.65-88.78)$ & 0.013 \\
\hline Urea nitrogen, $\mathrm{mmol} / \mathrm{L}^{\mathrm{a}}$ & $4.78(3.96-5.90)$ & $4.64(3.82-5.43)$ & $5.12(4.08-6.6)$ & 0.003 \\
\hline $\mathrm{UA}$ (uric acid), umol/ $\mathrm{L}^{\mathrm{a}}$ & $269.00(209.00-328.00)$ & $255.5(209.0-293.75)$ & $286(208-344.5)$ & 0.034 \\
\hline $\mathrm{D}$-dimer, $\mathrm{mg} / \mathrm{L}$ & $0.69(0.37-1.79)$ & $0.79(0.34-2.50)$ & $0.69(0.38-1.64)$ & 0.823 \\
\hline PT, s & $12.84(12.2-13.70)$ & $13.02(12.27-13.64)$ & $12.79(12.12-13.93)$ & 0.931 \\
\hline \multicolumn{5}{|l|}{ Electrolyte, $\mathrm{mmol} / \mathrm{L}$} \\
\hline $\mathrm{Na}^{+\mathrm{a}}$ & $141.60(139.20-143.40)$ & $141.4(138.65-143.40)$ & $141.7(139.35-143.33)$ & 0.726 \\
\hline $\mathrm{Cl}^{-\mathrm{a}}$ & $105.20(102.75-107.48)$ & $105.5(103.58-107.63)$ & $104.85(102.48-107.3)_{-}$ & 0.268 \\
\hline $\mathrm{K}^{+}$ & $4.33(3.98-4.65)$ & $4.4(4.1-4.77)$ & $4.2(3.83-4.57)$ & 0.002 \\
\hline $\mathrm{Ca}^{2-}$ & $2.09(2.00-2.16)$ & $2.11(2.02-2.17)$ & $2.07(1.99-2.16)$ & 0.154 \\
\hline \multicolumn{5}{|l|}{ Radiographic findings } \\
\hline Ground-glass opacity & $209(74.1)$ & $90(63.8)$ & $119(84.4)$ & $<0.001$ \\
\hline Consolidation & $18(6.4)$ & $3(2.1)$ & $15(10.6)$ & 0.004 \\
\hline Mottling opacity & $205(72.7)$ & $78(55.3)$ & $127(90.1)$ & $<0.001$ \\
\hline Bilateral & $271(96.1)$ & $136(96.5)$ & $135(95.7)$ & 0.756 \\
\hline Unilateral & $8(2.8)$ & $3(2.1)$ & $5(3.6)$ & 0.727 \\
\hline Unknown & $1(0.4)$ & $1(0.4)$ & 0 & \\
\hline Normal & $2(0.7)$ & $1(0.7)$ & $1(0.7)$ & \\
\hline Percentage of PIV, median (IQR), \% & $5.10(1.30-12.10)$ & $4.55(1.15-11.78)$ & $5.8(1.3-12.3)$ & 0.017 \\
\hline
\end{tabular}

[19]. Compared with healthy cases, monocytes from patients with essential hypertension are preactivated and would produce more IL-6 after stimulation with angiotensin II or lipopolysaccharide [20]. The immune dysregulation in patients with hypertension makes the cases to be more prone to be infected with SARS-CoV-2. In addition, in humans, ACE2 is expressed widely in many organs, including the heart, kidney, livers, intestines, and the lung alveolar epithelial cells [19]. However, it was found that $83 \%$ of ACE2expressing cells are type II alveolar cells [21] and the large surface area of the lung makes SARS-CoV-2 particularly vulnerable to inhaled viruses. Therefore, it is reasonable that
SARS-CoV-2 was more likely to damage the lung tissue of patients with hypertension, leading to increased numbers of severe cases.

In addition, it was found that neutrophil count patients and the level of $\mathrm{K}^{+}$were risk factors for COVID-19 in patients with hypertension. Neutrophils are a well-known marker of systemic inflammation and infection, usually as a predictor of bacterial infection. COVID-19-induced NKG2A expression may be correlated with functional exhaustion of lymphocytes (including CTLs and NK cells), which may result in severe pulmonary inflammation [22]. Because of the serious disturbance in the immune system, 
TABLE 3: Antihypertensive treatments of patients with COVID-19 and hypertension.

\begin{tabular}{|c|c|c|c|c|}
\hline & All $(n=151)$ & Nonsevere patients $(n=106)$ & Severe patients $(n=45)$ & $p$ value \\
\hline \multicolumn{5}{|l|}{ Classification of etiology } \\
\hline Primary & $149(98.7)$ & $104(98.1)$ & $45(100)$ & 0.881 \\
\hline Secondary & $2(1.3)$ & $2(1.9)$ & 0 & \\
\hline $\begin{array}{l}\text { Duration of hypertension, median (IQR), years } \\
\text { Grade }\end{array}$ & $10(6-20)$ & $10(6-13)$ & $15(9-22.5)$ & 0.065 \\
\hline 1 & $11(7.3)$ & $10(9.4)$ & $1(2.2)$ & 0.175 \\
\hline 2 & $34(22.5)$ & $21(19.8)$ & $13(28.9)$ & 0.222 \\
\hline 3 & $20(13.2)$ & $15(14.2)$ & $5(11.1)$ & 0.614 \\
\hline Unknown & $88(58.3)$ & $60(56.6)$ & $28(62.2)$ & 0.522 \\
\hline \multicolumn{5}{|l|}{ Antihypertensive therapies previously } \\
\hline CCBs & $85(56.3)$ & $61(57.5)$ & $24(53.3)$ & 0.094 \\
\hline ACEIs/ARBs & $30(19.9)$ & $26(24.5)$ & $4(8.9)$ & 0.031 \\
\hline ACEIs & $9(6.0)$ & $6(5.7)$ & $3(6.7)$ & 0.811 \\
\hline ARBs & $21(13.9)$ & $20(18.9)$ & $1(2.2)$ & 0.007 \\
\hline$\beta$-Blockers & $19(12.6)$ & $15(14.2)$ & $4(8.9)$ & 0.373 \\
\hline Anticoagulant drugs & $16(10.6)$ & $13(12.3)$ & $3(6.7)$ & 0.238 \\
\hline Statins & $10(6.6)$ & $7(6.6)$ & $3(6.7)$ & 0.988 \\
\hline None & $19(12.6)$ & $12(11.3)$ & $7(15.6)$ & 0.473 \\
\hline Diuretics & $7(4.6)$ & $4(3.8)$ & $3(6.7)$ & 0.426 \\
\hline Unknown & $14(9.3)$ & $9(8.5)$ & $5(11.1)$ & 0.76 \\
\hline
\end{tabular}

IQR: interquartile range; CCBs: calcium channel blockers; ACEIs: angiotensin-converting enzyme inhibitors; ARBs: angiotensin II type 1 receptor blockers; anticoagulant drugs: aspirin or clopidogrel.

TABLE 4: Univariate logistic regression analysis of patients with COVID-19 and hypertension.

\begin{tabular}{lcc}
\hline & Univariate OR (95\% CI) & $p$ value \\
\hline Diabetes & $2.148(1.074-4.297)$ & 0.031 \\
Coronary heart disease & $3.009(1.344-6.733)$ & 0.007 \\
Cerebrovascular disease & $4.279(1.181-15.510)$ & 0.027 \\
Percentage of PIV, \% & $1.025(1.004-1.046)$ & 0.021 \\
White blood cell count, $\times 109 / \mathrm{L}$ & $1.133(1.035-1.241)$ & 0.007 \\
Neutrophil count, $\times 109 / \mathrm{L}$ & $1.128(1.032-1.232)$ & 0.008 \\
ALP, IU/L & $1.007(0.998-1.016)$ & 0.112 \\
Urea nitrogen, mmol/L & $1.142(1.037-1.257)$ & 0.007 \\
UA (uric acid), umol/L & $1.003(1-1.005)$ & 0.028 \\
Creatinine, umol/L & $1.016(1.004-1.027)$ & 0.008 \\
$\mathrm{~K}^{+}$ & $0.582(0.383-0.885)$ & 0.011 \\
\hline
\end{tabular}

ALT: alanine aminotransferase; OR: odds ratio; CI: confidence interval.

TABle 5: Multivariate logistic regression analysis of patients with COVID-19 and hypertension.

\begin{tabular}{lcc}
\hline & Multivariate OR (95\% CI) & $p$ value \\
\hline Diabetes & $2.438(0.824-7.210)$ & 0.107 \\
Coronary heart disease & $5.281(1.462-19.076)$ & 0.011 \\
Cerebrovascular disease & $5.661(0.952-33.662)$ & 0.057 \\
Percentage of PLV, $\%$ & $0.994(0.963-1.026)$ & 0.71 \\
Neutrophil count, $\times 10^{9} / \mathrm{L}$ & $1.471(1.183-1.828)$ & 0.001 \\
Urea nitrogen, $\mathrm{mmol} / \mathrm{L}$ & $0.994(0.795-1.244)$ & 0.961 \\
UA (uric acid), umol/L & $1.003(0.998-1.008)$ & 0.247 \\
Creatinine, umol/L & $1.018(0.994-1.004)$ & 0.149 \\
$\mathrm{~K}^{+}$ & $0.273(0.134-0.555)$ & $<0.001$ \\
\hline
\end{tabular}

OR, odds ratio; CI, confidence interval.

which may also have an effect on the level of $\mathrm{K}^{+}$, cases with hypertension would get more susceptible to bacterial infection with neutrophils increased reactively and developed a potentially severe condition.

\section{Study Limitations}

There are some limitations to our study. First, given the retrospective design at a single center, not all laboratory tests 
TABLE 6: Univariate and multivariate logistic regression analysis of more severe patients with COVID-19 and hypertension.

\begin{tabular}{lccc}
\hline & Univariate OR $(95 \% \mathrm{CI})$ & $p$ value & Multivariate OR $(95 \% \mathrm{CI})$ \\
\hline Percentage of PIV, \% & $1.093(1.049-1.138)$ & $<0.001$ & $1.084(1.035-1.135)$ \\
$\mathrm{ARB}$ or ACEI & $0.299(0.097-0.923)$ & 0.036 & \\
$\mathrm{CK}-\mathrm{MB}, \mathrm{IU} / \mathrm{L}$ & $1.141(1.052-1.237)$ & 0.001 & \\
Age, years & $1.061(1.018-1.105)$ & 0.005 & \\
$\mathrm{Ca}^{2+}, \mathrm{mmol} / \mathrm{L}$ & $0.042(0.002-0.704)$ & 0.028 & \\
Platelet count, $\times 10^{9} / \mathrm{L}$ & $0.995(0.991-0.999)$ & 0.01 & \\
\hline
\end{tabular}

Blank cells indicate no statistical significance. OR: odds ratio; CI: confidence interval; PIV: pulmonary infection volume; $\mathrm{Ca}^{2+}$ : calcium ion; ACEI: angiotensin-converting enzyme inhibitors; ARB: angiotensin II type 1 receptor blockers.

were done in all patients and some records of the patients' medical history were missing. Second, there were about 33 from 282 patients with COVID- 19 and 28 from 151 COVID19 cases with hypertension were so severe that they were not able to complete a chest CT. The majority of the above patients had been taken chest radiographs bedside. In multivariable analysis, only cases with chest CT were included. Therefore, the value of the infection volume percentage might be underestimated in severe cases. Third, the sample size of the present study was small; larger populations and multiple centers are warranted to further confirm the outcomes of COVID-19 infection in patients with hypertension.

\section{Conclusions}

Coronary heart disease, neutrophil count patients, and the level of $\mathrm{K}^{+}$were associated with COVID-19 patients with hypertension. The percentage of the pulmonary infection volume was significantly larger in COVID-19 patients with hypertension and was a risk factor for COVID-19 severity of patients with hypertension. Early treatments should focus on lung protection for these cases and prevent bacterial infections if necessary.

\section{Data Availability}

The data in the study are available from the corresponding authors on reasonable request.

\section{Ethical Approval}

All procedures performed in studies involving human participants were in accordance with the ethical standards of the institutional and/or national research committee and with the 1964 Helsinki Declaration and its later amendments or comparable ethical standards.

\section{Consent}

Written or oral informed consent was waived.

\section{Conflicts of Interest}

The authors declare no conflicts of interest.

\section{Authors' Contributions}

Xinxin Wang and Haihua Zhang contributed equally to this work, extracted and analyzed the data, and drafted the manuscript. Tao Zhang and Yandong Nan designed and evaluated the quality of the study. Huan Du and Ruina Ma participated in the extraction and statistical analysis of the data.

\section{Acknowledgments}

The authors thank Haitao Zhang, Wei Liu, Hua Jiang, Chunmei Li, Yandong Nan, Hongjun Zhang, Wangping Li, Yanyan Li, and Shaokang Dang, for assistance with data collection. They also thank students of Air Force Medical University for providing assistance in data extraction.

\section{Supplementary Materials}

Supplementary Table 1: classification of office blood pressure and definitions of hypertension grades according to the 2018 guidelines of the European Society of Hypertension. Supplementary Table 2: reference range of laboratory findings. Supplementary Table 3: univariate logistic regression analysis of more severe patients with COVID-19 and hypertension. (Supplementary Materials)

\section{References}

[1] D. Wang, B. Hu, C. Hu et al., "Clinical characteristics of 138 hospitalized patients with 2019 novel coronavirus-infected pneumonia in Wuhan, China," JAMA, vol. 323, no. 11, p. 1061, 2020.

[2] C. Huang, Y. Wang, X. Li et al., "Clinical features of patients infected with 2019 novel coronavirus in Wuhan, China," The Lancet, vol. 395, no. 10223, pp. 497-506, 2020.

[3] Epidemiology Working Group for NCIP Epidemic Response, "Chinese center for disease control and prevention. The epidemiological characteristics of an outbreak of 2019 novel coronavirus diseases (COVID-19) in China," Zhonghua Liu Xing Bing Xue Za Zhi, vol. 41, pp. 145-151, 2020.

[4] Novel Coronavirus Pneumonia Emergency Response Epidemiology Team, "The epidemiological characteristics of an outbreak of 2019 novel coronavirus diseases (COVID-19) in China," Zhong Hua Liu Xing Bing Xue Za Zhi, vol. 41, pp. 145-151, 2020.

[5] E. Rodilla, A. Saura, I Jiménez et al., "Association of hypertension with all-cause mortality among hospitalized patients 
with COVID-19," Journal of Clinical Medicine, vol. 9, no. 10, p. E3136, 2020.

[6] M. Hoffmann, H. Kleine-Weber, S. Schroeder et al., "SARSCoV-2 cell entry depends on ACE2 and TMPRSS2 and is blocked by a clinically proven protease inhibitor," Cell, vol. 181, no. 2, pp. 271-280, 2020.

[7] R. Sommerstein and C. Gräni, "Rapid response: re: preventing a covid-19 pandemic: ACE inhibitors as a potential risk factor for fatal Covid-19," BMJ, vol. 19, 2020, http://www.bmj.com/ content/368/bmj.m810/rr-2.

[8] National Health Committee of the People's Republic of China, Chinese Clinical Guideline for COVID-19 Diagnosis and Treatment, National Health Committee, Beijing, China, 2020, http://www.nhc.gov.cn/yzygj/s7653p/202002/ 3b09b894ac9b4204a79db5b8912d4440.shtml, 5th edition.

[9] B. Williams, G. Mancia, W. Spiering et al., "2018 practice guidelines for the management of arterial hypertension of the European society of cardiology and the European society of hypertension," Blood Pressure, vol. 27, no. 6, pp. 314-340, 2018.

[10] H. Zhang, J. Zhang, and H. Zhang, "Automated detection and quantification of COVID-19 pneumonia: CT imaging analysis by a deep learning-based software," European Journal of Nuclear Medicine and Molecular Imaging, vol. 97, pp. 1-8, 2020.

[11] M. Singer, C. S. Deutschman, C. W. Seymour et al., "The third international consensus definitions for sepsis and septic shock (Sepsis-3)," JAMA, vol. 315, no. 8, pp. 801-810, 2016.

[12] F. Zhou, T. Yu, R. Du et al., "Clinical course and risk factors for mortality of adult inpatients with COVID-19 in Wuhan, China: a retrospective cohort study," The Lancet, vol. 395, no. 10229, pp. 1054-1062, 2020.

[13] X. Li, S. Xu, M. Yu et al., "Risk factors for severity and mortality in adult COVID-19 inpatients in Wuhan," Journal of Allergy and Clinical Immunology, vol. 146, no. 1, pp. 110$118,2020$.

[14] L. J. Burchill, E. Velkoska, R. G. Dean, K. Griggs, S. K. Patel, and L. M. Burrell, "Combination renin-angiotensin system blockade and angiotensin-converting enzyme 2 in experimental myocardial infarction: implications for future therapeutic directions," Clinical Science, vol. 123, no. 11, pp. 649-658, 2012.

[15] I. Hamming, W. Timens, M. Bulthuis, A. Lely, G. Navis, and H. van Goor, "Tissue distribution of ACE2 protein, the functional receptor for SARS coronavirus. A first step in understanding SARS pathogenesis," The Journal of Pathology, vol. 203, no. 2, pp. 631-637, 2004.

[16] Z. Li, M. Wu, and J. Yao, "Caution on kindney dysfunctions of COVID19 patients," Med.Rxiv., vol. 1, 2020, https://ssrn. com/abstract $=3559601$.

[17] X. Liu, H. Zhou, Y. Zhou et al., “Temporal radiographic changes in covid-19 patients: relationship to disease severity and viral clearance," Scientific Reports, vol. 10, no. 1, Article ID 10263, 2020.

[18] M. Yuan, W. Yin, Z. Tao, W. Tan, and Y. Hu, "Association of radiologic findings with mortality of patients infected with 2019 novel coronavirus in Wuhan, China," PLoS ONE, vol. 15, no. 3, Article ID e0230548, 2020.

[19] H. A. Itani, W. G. McMaster, M. A. Saleh et al., "Activation of human T cells in hypertension," Hypertension, vol. 68, no. 1, pp. 123-132, 2016.

[20] D. Yvonne, "Preactivated peripheral blood monocytes in patients with essential hypertension," Hypertension, vol. 34, pp. 113-117, 1999.
[21] Y. Zhao, Z. Zhao, Y. Wang, Y. Zhou, Y. Ma, and W. Zuo, "Single-cell RNA expression profiling of ACE2, the putative receptor of Wuhan 2019-nCov," BioRxiv, vol. 26, 2020.

[22] M. Zheng, Y. Gao, and G. Wang, "Functional exhaustion of antiviral lymphocytes in COVID-19 patients," Cellular \& Molecular Immunology, vol. 17, no. 5, 2020. 